Boise State University

ScholarWorks

8-16-2012

Evaluation of an Internet-Short Message Service-Based Intervention for Promoting Physical Activity in Hong Kong Chinese Adolescent School Children: A Pilot Study

Erica Y. Lau

University of South Carolina - Columbia

Patrick W. C. Lau

Hong Kong Baptist University

Pak-Kwong Chung

Hong Kong Baptist University

Lynda B. Ransdell

Boise State University

Edward Archer

University of South Carolina - Columbia 


\title{
Evaluation of an Internet-Short Message Service-Based Intervention for Promoting Physical Activity in Hong Kong Chinese Adolescent School Children: A Pilot Study
}

\author{
Erica Y. Lau, M.Phil., ${ }^{1}$ Patrick W.C. Lau, Ph.D., ${ }^{2}$ Pak-Kwong Chung, D.P.E., ${ }^{2}$ \\ Lynda B. Ransdell, Ph.D., ${ }^{3}$ and Edward Archer, M.S. ${ }^{1}$
}

\begin{abstract}
Evaluation of acceptability and preliminary efficacy of an Internet and short message service (SMS) intervention for promoting physical activity (PA) in Hong Kong Chinese school children. An 8-week quasi-experimental study non-randomly assigned 78 school children (mean age $=12.8$ years) to (a) an intervention group that received a stage-matched, Internet PA program two times a week and tailored SMS messages daily; or (b) a notreatment control. Data were collected from September 2008 until June 2009. Acceptability measures included exposure rate and participant's satisfaction. Efficacy measures were changes in stage of motivational readiness (SMR) and self-reported PA level. Intervention participants demonstrated significant pre-post increments in SMR $(Z=-2.558, p=0.011)$ and self-reported PA level $[F(1,76)=4.50, p=0.04]$. There was a non-significant trend between groups in both SMR $(p=0.24)$ and PA $(p=0.13)$. Despite the similar ratings of satisfaction between Internet $(M=3.12 \pm 0.74)$ and SMS $(M=3.12 \pm 0.84)$, participants displayed distinct patterns of exposure with $66 \%$ exhibiting a weekly login rate of 0.5 times/person and an average of 3.75 minutes/visit/person. In contrast, $79 \%$ of participants read an average of $1.3 \mathrm{SMS} /$ person/week and $47 \%$ voluntarily replied to $\sim 3.8 \mathrm{SMS} /$ person. These findings demonstrate the acceptability and preliminary efficacy of an Internet-SMS-based intervention for promoting PA in Hong Kong school children. The divergent exposure rates between the Internet and SMS may be a unique pattern for adolescents in early SMR. Future research should be cognizant of the importance of SMR since it may influence utilization and/or adherence.
\end{abstract}

\section{Introduction}

$\mathbf{R}$ EGULAR PHYSICAL ACTIVITY (PA) is associated with a reduced risk of non-communicable chronic diseases, such as diabetes and obesity, in adolescents. ${ }^{1,2}$ However, $<50 \%$ of adolescents in developed countries meet PA recommendations (i.e., $\geq 60$ minutes of moderate-to-vigorous PA on most days of the week). ${ }^{3-6}$ Given the consequences and prevalence of adolescent physical inactivity, it is essential to develop efficacious interventions for promoting PA.

Information and Communication Technologies (ICTs), and particularly the Internet and mobile phones, are often advocated as an important medium for health communication and intervention in youth due to its ease of use, high accessibility, and acceptability. ${ }^{7-10}$ In developed countries (e.g., United States and Hong Kong), over $90 \%$ of the adolescent population has access to Internet ${ }^{6,11}$ and uses it regularly for activities that range from learning to social networking and shopping. ${ }^{12}$ A majority of adolescents own a mobile phone and $50 \%$ are users of short message services (SMSs) (i.e., text messaging). ${ }^{13}$ Previous research has shown that $70 \%$ of adolescents have used the Internet to obtain health-related information $^{14,15}$ and many of them have a positive attitude toward ICT delivered interventions. ${ }^{16,17}$ The ease of use and compatibility with adolescent's lifestyle ${ }^{18}$ may increase the likelihood of productive behavior change via enhanced adherence. ${ }^{19,20}$ As such, ICT modalities may provide an effective medium in which to promote PA and reduce risk factors for disease. ${ }^{9,10}$

A systematic review evaluated the efficacy of ICT-based PA interventions for youth and found that more than $65 \%$ demonstrated positive effects on psychosocial (e.g., social support) or behavioral (e.g., step counts) PA outcomes. ${ }^{21}$ The key characteristics of effective ICT-based interventions ${ }^{21-23}$ are grounding in theory and use of behavior change techniques. ${ }^{24-27}$ Moreover, the combined use of Internet and SMS

\footnotetext{
${ }^{1}$ Department of Exercise Science, Arnold School of Public Health, University of South Carolina, Columbia, South Carolina.

${ }^{2}$ Department of Physical Education, Hong Kong Baptist University, Kowloon Tong, Hong Kong.

${ }^{3}$ Department of Kinesiology, College of Education, Boise State University, Boise, Idaho.
} 
(I-SMS) may produce more positive impacts than either modality alone. ${ }^{21,22}$ The proposed mechanism for the concomitant efficacy of the I-SMS approach is the autonomy afforded when viewing and selecting components. ${ }^{28}$ Additionally, the SMS utilizes a "push" strategy whereby researchers and health professionals deliver automatic, tailored information (e.g., medication compliance and performance feedback). Tailored messages may result in a greater propensity for behavior change as the information may be viewed as relevant and personalized. ${ }^{29}$

The dual-modality approach, I-SMS-based interventions, for PA behavior change in adolescents is still developing. ${ }^{21}$ Nonetheless, it has shown promising results in adult population. ${ }^{30,31}$ Since the I-SMS approach offers the possibility of a synergistic effect leading to enhanced information dissemination, its potential effect on adolescent's PA behavior merits investigation. The objectives of this pilot study were to evaluate the acceptability and preliminary efficacy of a theorybased, I-SMS-based intervention for promoting PA in Hong Kong Chinese school children.

\section{Methods}

An 8-week quasi-experimental study non-randomly assigned 78 school children (mean age $=12.8$ years) to intervention or control group. The intervention consisted of an Internet PA program accessed twice per week and daily SMS (i.e., texts) in weekdays. The control group received no intervention. Data were collected from September 2008 until June 2009. Measurements were obtained at baseline and postintervention.

\section{Description and selection criteria of participants}

A convenience sample of adolescent school children $(n=78)$ was recruited from three secondary schools in Hong Kong (Fig. 1). Inclusion/exclusion criteria were as follows: (a) 12-15 years old, (b) Internet access at home, (c) possess a personal mobile phone, (d) no physical or psychological limitation that precluded PA, and (e) currently not meeting $P A$ recommendation as determined by a staging algorithm ${ }^{32}$ (Table 1). All study procedures were approved by the Research Ethics Committee of Hong Kong Baptist University.

\section{Theoretical model}

The Stage of Motivational Readiness for Change (SOC) model $^{32,33}$ has been used successfully in PA interventions for adolescents ${ }^{21}$ and was used to guide the intervention design. The SOC model provides constructs that suggest why, when, and how people change: (a) stage of motivational readiness (SMR), (b) decisional balance (pros and cons), (c) self-efficacy (perceived confidence), and (d) processes of change (POC) (strategies for change). The SMR categorizes the timing for behavior change into five stages: (a) pre-contemplation (i.e., inactive and not thinking about becoming active), (b) contemplation (i.e., inactive but thinking about becoming active), (c) preparation (i.e., PA not at recommended levels), (d) action (i.e., PA at recommended levels $<6$ months), and (e) maintenance (i.e., PA at recommended level $>6$ months). Stage progression is posited to be linked with improvements in decisional balance and self-efficacy, ${ }^{32}$ and those who progress demonstrate increased utilization of the POC. ${ }^{34}$
Previous studies ${ }^{35,36}$ demonstrated that individuals in the early stages of change (SOC) were more likely to have lower levels of PA, decisional balance, self-efficacy, and use fewer POC than individuals in later stages. These results suggest that interventions that enhance decisional balance, selfefficacy, and/or use of POC may be effective in promoting PA in individuals in the early SOC. These stage-specific participant characteristics also facilitate intervention design via the tailoring of content and presentation.

\section{Study design}

An 8-week quasi-experimental trial in which, after schoollevel stratification, adolescent school children were assigned to an intervention or control group. The intervention group consisted of an Internet PA program and SMS. Intervention participants were directed to access the Internet PA program twice per week and read daily text messages. The control group did not receive any intervention.

The Internet PA program was designed to improve participant's PA behavior via the provision of stage-matched PA resources and self-monitoring tools. Texts were an adjunct mode of delivery to reinforce the Internet PA program (e.g., providing encouragement) (Fig. 2). To maintain adherence, monthly incentives (e.g., water bottles) were offered. Although responding to the SMS was optional, each participant was subsidized with HKD \$30 per month (each SMS costs HKD \$0.5-\$1.5). Recruitment began in September 2008 and data were collected until June 2009. Questionnaires were used to collect participant's demographics. Participant's height and weight were measured by school physical education (PE) teachers.

\section{Intervention components}

Internet PA program. The Internet PA program "Teenstep.com" (Appendix 1) was based on previously tested materials using the SOC model. ${ }^{32,37,38}$ "Teenstep.com" provides stage-matched information, behavioral skill training, and self-monitoring tools to facilitate PA behavior change in adolescents. Teenstep.com consists of four modules: (1) "Why Active?," (2) "Becoming Active," (3) "Set Goals!," and (4) "Keep Going!" Modules 1, 2, and 3 were designed for participants in the pre-contemplation, contemplation, and preparation stage, respectively. Module 4 was developed for adolescents who successfully transited from preparation to the action stage (Appendix 2). The interactive features of the Web site included tailored feedback, a PA planner, and chat room. Once logged onto the program, participants were directed to the Physical Activity Readiness Questionnaire $^{39}$ and a staging questionnaire ${ }^{32}$ for the stage matching (i.e., tailoring) of intervention modules. At the conclusion of each module, the participant's SMR was re-assessed and the system directed them to (a) progress to a new module or (b) remain in the current module.

A modified Delphi method ${ }^{40}$ was used to ensure literacy level and appropriateness of contents and materials. The Delphi participants consisted of three ICT intervention experts and five secondary school PE teachers. Moreover, a formative assessment was conducted. These preliminary procedures led to productive adjustments (i.e., reduced text volume and enlarged font size). 


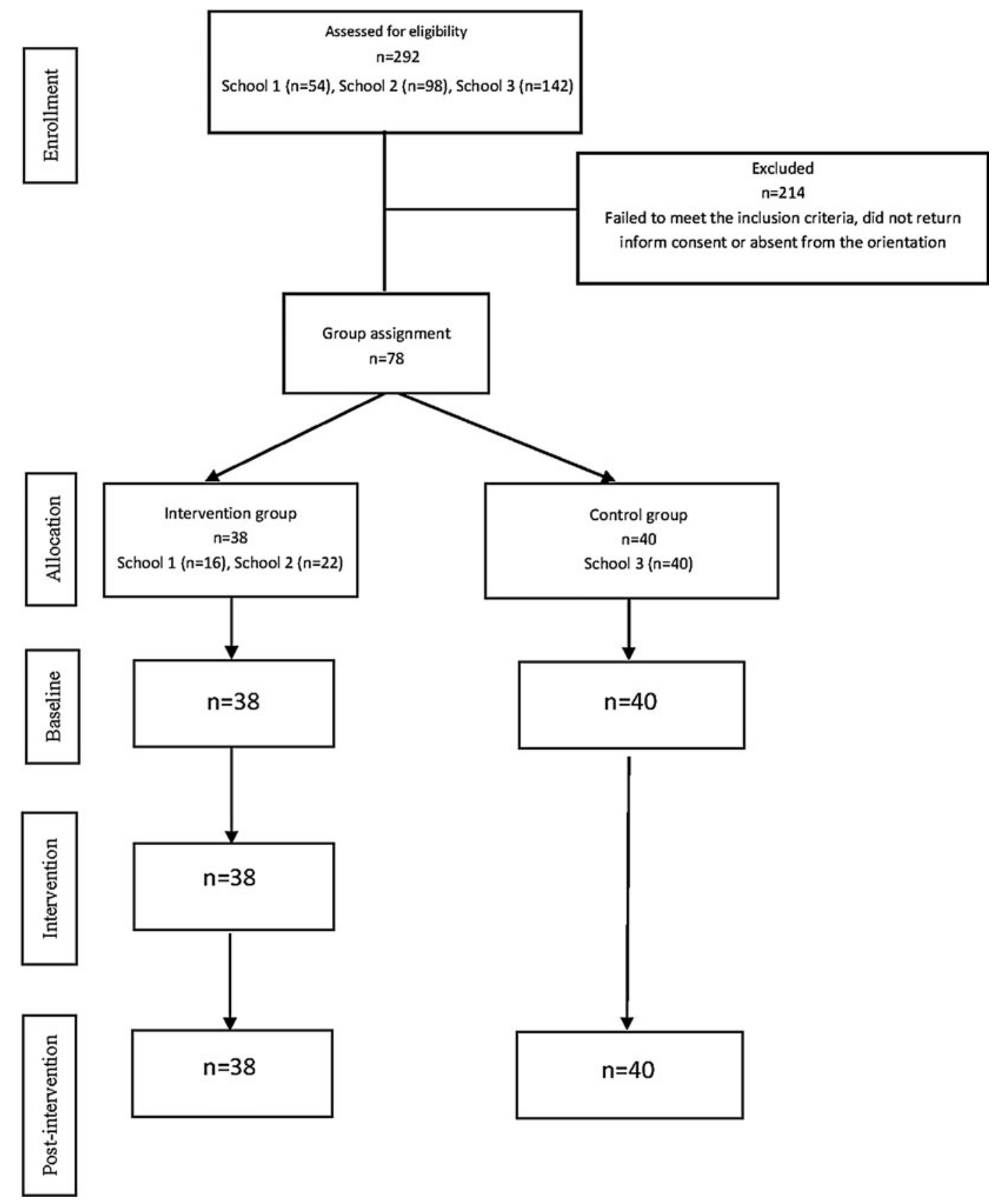

FIG. 1. Flow diagram of participants.

\section{Short message service}

The SMS was adapted from previous SMS-based health behavior interventions for adolescents ${ }^{41-44}$ and the SOC model. $^{32,33}$ The SMS was constructed to imitate a colloquial dialogue (Cantonese) with a virtual friend "Jackie," whose purpose was to establish rapport and make the intervention similar to "human-to-human" interactions. Previous studies $^{41-44}$ suggest that adolescents perceive peer-to-peer dialogue as more acceptable than messages from a health professional. The SMS consisted of five message types: (a) motivational, (b) informational, (c) behavioral skills, (d) reinforcement of PA benefits, and (e) solutions for PA barriers (Appendix 3 for sample SMS messages).

\section{Procedures}

Invitation letters were sent to multiple Hong Kong secondary schools and three agreed to participate. Participants were recruited via flyers. Prescreening consisted of questionnaires on inclusion criteria (e.g., Internet access). A fouritem staging algorithm was used to determine SMR and PA behavior. Adolescents in pre-contemplation, contemplation, and preparation stages were invited to participate. Participants and their parents/guardians were informed of study procedures and they signed informed consent. An orientation provided participants with an online identity, and printed instructions on accessing the Internet PA program, and how to receive/respond to the SMS. An SMS was sent to the 
Table 1. Participant Characteristics at Baseline by Group (Mean \pm Standard Deviation)

\begin{tabular}{|c|c|c|}
\hline & $\begin{array}{l}\text { Control } \\
(n=40)\end{array}$ & $\begin{array}{l}\text { Intervention } \\
\quad(n=38)\end{array}$ \\
\hline Age $^{a}$ & $13.26 \pm 1.14$ & $12.29 \pm 0.87$ \\
\hline BMI & $18.99 \pm 3.22$ & $18.31 \pm 3.48$ \\
\hline Self-reported physical activity & $1.77 \pm 0.36$ & $1.85 \pm 0.27$ \\
\hline \multicolumn{3}{|l|}{ Sex } \\
\hline Male & $37.5 \%$ & $31.6 \%$ \\
\hline Female & $62.5 \%$ & $68.4 \%$ \\
\hline \multicolumn{3}{|c|}{ Internet usage frequency (days/week) ${ }^{\mathrm{a}}$} \\
\hline No & $35 \%$ & $7.9 \%$ \\
\hline 1-2 days & $32.5 \%$ & $21.1 \%$ \\
\hline 3-4 days & $12.5 \%$ & $15.8 \%$ \\
\hline $5-6$ days & $5 \%$ & $21.1 \%$ \\
\hline 7 days & $15 \%$ & $34.2 \%$ \\
\hline \multicolumn{3}{|c|}{ Internet usage duration (minutes/day) ${ }^{\mathrm{a}}$} \\
\hline$<10 \min$ & $0 \%$ & $15.8 \%$ \\
\hline $11-30 \mathrm{~min}$ & $27.5 \%$ & $18.4 \%$ \\
\hline $31-59 \mathrm{~min}$ & $10 \%$ & $15.8 \%$ \\
\hline $60-120 \mathrm{~min}$ & $22.5 \%$ & $18.4 \%$ \\
\hline$\geq 120 \mathrm{~min}$ & $40 \%$ & $31.6 \%$ \\
\hline \multicolumn{3}{|l|}{ Stage of motivational readiness } \\
\hline Pre-contemplation & $62.5 \%$ & $55.3 \%$ \\
\hline Contemplation & $30.0 \%$ & $34.2 \%$ \\
\hline Preparation & $7.5 \%$ & $10.5 \%$ \\
\hline
\end{tabular}

a Significant between-group differences at $p<0.05$.

BMI, body-mass index.

participants to remind them to start using the Internet PA program.

\section{Outcome measures}

Outcome measures for both groups were assessed at baseline and at the end of the intervention. PE teachers of participating schools arranged the venue and researchers administered the questionnaires.

\section{Acceptability measures}

Intervention exposure rate. Frequency and duration of exposure to the Internet PA program were measured. Exposure frequency was the number of times the participant accessed the program. Exposure duration was the number of minutes per visit. An embedded database tracked the hit rate of the Internet PA program and duration (i.e., minutes/ person for each visit). Exposure frequency, but not duration, was measured for the SMS. The numbers of SMS replied were recorded via a commercial database. At the completion of the intervention, participants also reported the number of SMS they read per week on a survey.

Satisfaction with the intervention. A custom questionnaire was developed to quantify and examine the participant's satisfaction with the Internet PA program (five items, Chronbach's $\alpha=0.92$ ) and SMS (four items, Chronbach's $\alpha=0.91)$ based on a five-point Likert scale ( $1=$ extremely disagree, $5=$ extremely agree). Participants also responded to two additional items with yes/no options: (a) "do you want to continue to receive the SMS motivators?" and (b) "would you recommend this I-SMS PA intervention to your friends?"

\section{Efficacy measures}

PA level. The nine-item Physical Activity Questionnaire for Older Children (PAQC) ${ }^{45}$ was employed to measure participant's PA level over the last 7 days (five-point Likert scale). The reliability for the PAQC is 0.83 and has been validated with the Godin and Shephard Leisure Time Exercise Questionnaire (total exercise score: $r=0.41$ ) and the SevenDay Recall Interview (hard activity: $r=0.31, p<0.05$; very hard activity: $r=0.42, p<0.05) .{ }^{46}$

Stage of motivational readiness. A staging algorithm ${ }^{32}$ was used to measure participant's SMR. The staging algorithm consisted of four yes/no option items to categorize participants into five stages: pre-contemplation, contemplation, preparation, action, and maintenance. The scale had a kappa index of reliability of 0.78 over a 2-week period and has been previously validated with the Seven-Day Physical Activity Recall. ${ }^{32}$

\section{Statistical analysis}

Baseline characteristics were compared using chi-square tests for categorical variables and independent $t$ tests for continuous variables. The data were analyzed on the intent-

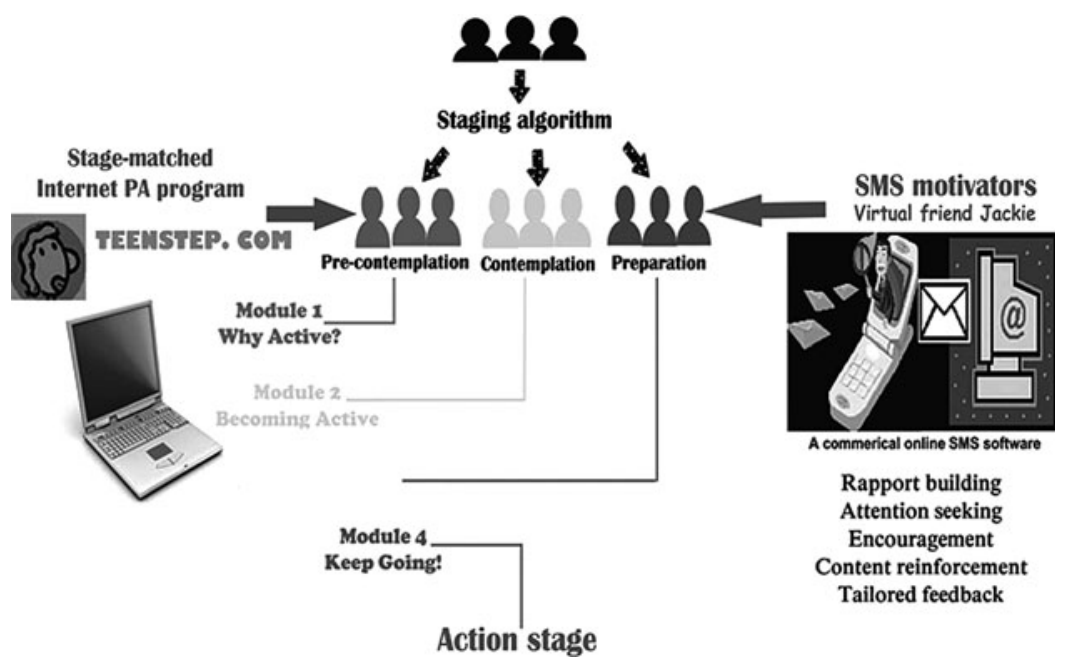

FIG. 2. Mechanism of the I-SMS-based intervention. SMS, short message service. 
Table 2. Participant's Satisfactory to the Internet Physical Activity Program and the Short Message Service (Mean \pm Standard Deviation)

\begin{tabular}{lr}
\hline Items & Total \\
\hline Web components (Chronbach's $\alpha=0.92)$ & $3.12 \pm 0.74$ \\
1. The web content is easy to understand & $3.21 \pm 0.87$ \\
2. The web content is relevant to me & $3.10 \pm 0.91$ \\
3. The web content is credible & $3.26 \pm 0.77$ \\
4. The web interface is well designed & $3.12 \pm 0.84 \pm 0.82$ \\
5. The web is easy to navigate & $3.12 \pm 0.86$ \\
SMS components (Chronbach's $\alpha=0.91)$ & $2.95 \pm 1.01$ \\
1. The SMS content can encourage me to do PA & $3.07 \pm 0.99$ \\
2. The SMS content is relevant to me & $3.31 \pm 0.87$ \\
3. The SMS content is easy to understand & $3.14 \pm 0.90$ \\
4. The language of the SMS is tailored for adolescents & Yes \\
Additional questions & $19 \%$ \\
1. Would you recommend this I-SMS PA intervention to your friends? & $59 \%$ \\
2. Do you want to continue to receive the SMS motivator? & $81 \%$ \\
\hline
\end{tabular}

$\mathrm{M}$, mean; SD, standard deviation; SMS, short message service; PA, physical activity.

to-treat principle and missing data were substituted by last observation carried forward method.

Descriptive statistics were used to analyze the intervention exposure rate. Partial correlations were used to examine (a) change in efficacy outcomes (i.e., self-reported PA and SMR) in relation to the Internet PA program mean logon rate while controlling for SMS exposure and (b) change in efficacy outcomes in relation to exposure to SMS while controlling for mean logon rate of the Internet PA program. A Wilcoxon signed rank test was performed to assess the change in baseline SMR. To compare stage movement between groups, participants were divided into three categories (i.e., stable, stage progression, and stage regression) and analyzed using a chi-square test. A $2 \times 2$ (treatment $\times$ time) repeated measures analysis of variance was conducted for the effect of treatment (intervention vs. control) and time (baseline and post-intervention) on PA level. Follow-up analyses were conducted when significant main effects were found. A post hoc power analysis was conducted using actual sample and effect size. All analyses were conducted using the SPSS statistical package (version 16.0; SPSS, Inc., Chicago, IL). The level of significance was defined as $p<0.05$ and all tests were two-tailed.

\section{Results}

All participants $(100 \%)$ completed the pre-test and posttest questionnaires.

\section{Acceptability}

All intervention participants logged onto the Internet PA program at least once with $66 \%$ revisiting multiple (i.e., $\geq 2$ ) times. Mean weekly login rate was 0.5 times/person. Total exposure duration for each participant was 30 minutes and average duration per visit was 3.75 minutes. Approximately $79 \%$ of participants read an average of $1.3 \mathrm{SMS} /$ person/week and $47 \%$ voluntarily replied to $\sim 3.8$ SMS/person. Participants were satisfied with the content and design with $81 \%$ affirming that they were willing to continue receiving the SMS and 59\% would recommend the I-SMS system to their friends (Table 2).

A moderate, positive relationship was found between number of SMS read and changes in SMR $(r=0.47, p=0.009)$.
An inspection of zero-order correlation $(r=0.46, p=0.01)$ suggested that controlling for exposure to Internet component (i.e., Internet PA program mean logon rate) had little effect on the strength of the relationship between the two variables.

\section{Preliminary efficacy}

Baseline and post-intervention efficacy outcomes are presented in Table 3. A significant improvement in SMR was observed in the intervention group $(Z=-2.558, p=0.011)$, but not in the control group $(Z=-0.996, p=0.32)$ (Table 3$)$. In contrast to controls, intervention participants demonstrated a higher percentage of stage progression (39.5\% vs. $22.5 \%$ ), with more participants advancing to preparation stage or beyond $(18.4 \%$ vs. $11 \%)$, and fewer participants were stage neutral (50\% vs. $60 \%)$ or regressed to an earlier stage $(10.5 \%$ vs. $17.5 \%$ ). Between-group differences were not statistically significant $\left[\chi^{2}(2,78)=2.85, p=0.24\right]$. There was a significant main effect for time $\left[F(1,76)=4.50, p=0.04\right.$; partial $\left.\eta^{2}=0.06\right]$ in self-reported PA. Follow-up analysis (paired-sample $t$ test) indicated a significant increase in self-reported PA in the intervention group $[t(37)=2.07, p=0.05$; mean change $=0.19 \pm$ $0.53]$ versus control group $[t(39)=0.10, p=0.34$; mean change $=0.09 \pm 0.59$ ]. There was a non-significant trend for

Table 3. Participant's Measured Outcomes at Baseline and Post-Intervention by Group

\begin{tabular}{lcc}
\hline & $\begin{array}{c}\text { Control } \\
(n=40)\end{array}$ & $\begin{array}{c}\text { Intervention } \\
(n=38)\end{array}$ \\
\hline PAQC $(M \pm S D)$ & & \\
Baseline & $1.77 \pm 0.36$ & $1.85 \pm 0.27$ \\
Post-intervention & $1.85 \pm 0.63$ & $2.03 \pm 0.52^{\mathrm{a}}$ \\
SMR (median) & & \\
Baseline & 1.0 & 1.0 \\
Post-intervention & 1.0 & $2.0^{\mathrm{a}}$ \\
\hline
\end{tabular}

${ }^{a}$ Significant within-group differences at $p<0.05$.

PAQC, Physical Activity Questionnaire for Older Children; SMR, stage of motivational readiness. 
interaction effects (group $\times$ time) $[F(1,76)=0.53, p=0.47]$ and main effects for group $[F(1,76)=2.41, p=0.13]$.

\section{Discussion}

The present study provides support for the acceptability and preliminary efficacy of I-SMS-based PA interventions on Hong Kong school children's PA behavior. Intervention participants demonstrated pre-post increments in SMR and self-reported PA. Approximately $81 \%$ affirmed that they were willing to continue receiving the SMS and 59\% would recommend the I-SMS system to their friends. While intervention participants expressed similar levels of satisfaction for the Internet PA program and the SMS, the exposure rate varied substantially. Only $66 \%$ of participants logged onto the Internet program greater than or equal to two times, and the majority of those were less than three times over the 8 -week study. The weekly logon rate for each participant was 0.5 times that was lower than intended rate of two times. The maintenance of an adequate exposure rate is challenging in Internet-based behavior change interventions. It has been suggested that the participant's perception of the necessity for behavior change may be a critical characteristic that determines exposure rate. ${ }^{47,48}$ Other stage models have classified those perceived low necessity to change (i.e., precontemplators and contemplators) as pre-intenders. ${ }^{49}$ Typical characteristics of pre-intenders are lower levels of motivation, self-efficacy, and poor behavior change skills. ${ }^{32,33}$ These characteristics make them more likely to avoid cognitively demanding tasks. ${ }^{29}$ Unfortunately, an interactive Internet program may be viewed as demanding given the interactive nature of the program (e.g., participants have to continuously specify preferences via clicking the mouse).$^{50}$ Although previous studies suggest that interactivity is a key element for increasing Web site exposure rates, ${ }^{50,51}$ it may be more effective with those individuals in the later SOC. The cognitive burden of interactivity may impede adherence and participation of pre-intenders.

Contrary to the Internet PA program, $\sim 79 \%$ of the participants read $\geq 1$ SMS/week and about half of them responded. The number of SMS read positively correlated with the change in SMR after controlling for exposure to the Internet PA program. These findings indicate that "pushed" health information ${ }^{52}$ may be more effective with pre-intenders. Accordingly, the Technology Acceptance model ${ }^{53}$ suggests that interventions perceived as effortless will be more likely to report a higher exposure rate.

To our knowledge, this is the first I-SMS intervention to report the exposure rates of Internet and SMS components independently as well as their contribution to efficacy. Our results suggest that there may be an interaction between the different modes of information dissemination and SOC. For example, personalized SMS alone requires little effort and therefore may be more effective for individuals in the early SOC. In contrast, a comprehensive and interactive Internet PA program may better meet the needs of individuals in the latter SOC since they take an active role in obtaining information. ${ }^{32,33}$ Nevertheless, future investigations should include objective measures of exposure.

The current study did not detect significant between-group effect or interaction effect. However, it is important to note that the increment in SMR and self-reported PA in the inter- vention group achieved medium effect sizes. A lack of power was responsible for the lack of significant between-group differences in that previous studies have shown significant between-group difference when medium effect sizes are achieved. ${ }^{21}$ The results of the post hoc power analysis indicate that a sample size much greater than that of our pilot study (i.e., $\geq 126$ ) would be required to detect a significant betweengroup and interaction effect $(1-\beta=80 \%$, effect size $d=0.5$, and Chronbach's $\alpha=0.05)$. This finding provides valuable information for future randomized controlled trials.

The present study has a number of strengths, and the positive findings demonstrate the necessity of theory-based intervention design, while providing support for the use of SOC constructs for tailoring ICT-based PA interventions. Objective measures for frequency and/or duration of intervention exposure rates for both modes are a significant advancement in our understanding of the potential for an interaction between the mode of information delivery and the individual's SOC. Our findings offer novel insights on technology selection and suggest that future investigators examine combinations of technologies to optimize respective strengths and minimize the limitations engendered by the SOC characteristics of diverse populations. ${ }^{54,55}$

In conclusion, this pilot study demonstrated that a stagematched, I-SMS-based intervention is an acceptable approach for promoting PA among Hong Kong school children and provides support for the use of SOC constructs in the design and implementation of I-SMS-based interventions.

\section{Acknowledgments}

The authors would like to thank all the participants and school teachers for their participation and cooperation.

\section{Author Disclosure Statement}

No competing financial interests exist.

\section{References}

1. Hallal PC, Victora CG, Azevedo MR, et al. Adolescents physical activity and health. A systematic review. Sports Medicine 2006; 36:1019-1030.

2. Warburton DE, Nicol CW, Bredin SS. Health benefits of physical activity: the evidence. Canadian Medical Association Journal 2006; 174:801-809.

3. Brodersen NH, Steptoe A, Boniface DR, et al. Trends in physical activity and sedentary behaviour in adolescence: ethnic and socioeconomic differences. British Journal of Sports Medicine 2007; 41:140-144.

4. Janssen I, Katzmarzyk PT, Boyce WF, et al. Comparison of overweight and obesity prevalence in school-aged youth from 34 countries and their relationships with physical activity and dietary patterns. Obesity Reviews 2005; 6:123-132.

5. Hamar P, Biddle S, Soos I, et al. The prevalence of sedentary behaviours and physical activity in Hungarian youth. European Journal of Public Health 2010; 20:85-90.

6. Koezuka N, Koo M, Allison KR, et al. The relationship between sedentary activities and physical inactivity among adolescents: results from the Canadian community health survey. International Journal of Adolescent Medicine and Health 2006; 39:515-522.

7. PREVE. White paper-ICT research direction in disease prevention 2011. www.preve-eu.org/ (accessed Feb. 20, 2012). 
8. US Department of Health and Human Services. Healthy people 2020 summary of objectives. www.healthypeople .gov/2020/Consortium/HP2020Framework.pdf (accessed May 19, 2011).

9. US Department of Health and Human Services. Expanding the reach and impact of consumer e-health tools (pdf). www.health.gov/communication/ehealth/ehealthtools/pdf/ ehealthreport.pdf (accessed Feb. 20, 2012).

10. US Department of Health and Human Services. National physical activity plan for the United States. www.physical activityplan.org/media.php (accessed Feb. 20, 2012).

11. Lenhart A, Purcell K, Smith A, et al. (2010) Social media and mobile Internet use among teens and young adults. Pew Research Center.

12. Lenhart A, Madden M, Hitlin P. (2005) Teens and technology. Pew Research Center.

13. Treweek S. Joining the mobile revolution. Scandinavian Journal of Primary Health Care 2003; 21:75-76.

14. Hansen DL, Derry HA, Resnick PJ, et al. Adolescents searching for health information on the Internet: an observational study. Journal of Medical Internet Research 2003; 5(4):e25.

15. Santor DA, Poylin C, LeBlance JC, et al. Online health promotion, early identification of difficulties, and help seeking in young people. Journal of the American Academy of Child and Adolescent Psychiatry 2007; 46:50-59.

16. Tercyak KP, Abraham AA, Graham AL, et al. Association of multiple behavioral risk factors with adolescents willingness to engage in eHealth promotion. Journal of Pediatric Psychology 2009; 34:457-469.

17. Tate DF. (2009) Application of innovative technologies in the prevention and treatment of overweight in children and adolescents. Springer.

18. Vincent J. (2005) Emotional attachment and mobile phone. In Glotz P, Bertschi S, Locke C, eds. Thumb culture. The meaning of mobile phone for society. Bielefied: Transcript Verlag.

19. Roger E. (2003) Diffusion of innovation. New York: Free Press.

20. Chan CV, Kaufman DR. A technology selection framework for supporting delivery of patient-oriented health interventions in developing countries. Journal of Biomedical Informatics 2010; 43:300-306.

21. Lau PWC, Lau EY, Wong DP, et al. A systematic review of ICT-based interventions for promoting physical activity behavior change in children and adolescents. Journal of Medical Internet Research 2011; 13:e48.

22. Norman GJ, Zabinski MF, Adam MA, et al. A review of eHealth interventions for physical activity and dietary behavior change. American Journal of Preventive Medicine 2007; 33:336-345.

23. Fjeldsoe BS, Marshall AL, Miller YD. Behavior change interventions delivered by mobile telephone short message service. American Journal of Preventive Medicine 2009; 36: 165-173.

24. Webb TL, Joseph J, Yardley L, et al. Using the Internet to promote health behavior change: a systematic review and meta-analysis of the impact of theoretical basis, use of behavior change techniques, and mode of delivery on efficacy. Journal of Medical Internet Research 2010; 12:e4.

25. Cushing CC, Steele RG. A meta-analytic review of eHealth interventions for pediatric health promotion and maintaining behaviors. Journal of Pediatric Psychology 2010; 35:937949.

26. Portnoy DB, Scott-Sheldon LAJ, Johnson BT, et al. Computer-delivered interventions for health promotion and be- havioral risk reduction: a meta-analysis of 75 randomized controlled trials, 1988-2007. Preventive Medicine 2008; 47: 3-16.

27. Lustria MLA, Cortese J, Noar SM, et al. Computer-tailored health interventions delivered over the web: review and analysis of key components. Patient Education and Counseling 2009; 74:156.

28. Deci EL, Ryan RM. The 'what' and 'why' of gal pursuits: human needs and the self-determination of behavior. Psychological Inquiry 2000; 11:227-268.

29. Petty RE, Cacioppo JT. (1986) Communication and persuasion, central and peripheral routes to attitude change. New York: Springer.

30. Hurling R, Fairley BW, Dias MB. Internet-based exercise intervention systems: are more interactive designs better? Psychology and Health 2006; 21:757-772.

31. Hurling R, Catt M, Boni MD, et al. Using Internet and mobile phone technology to deliver an automated physical activity program: randomized controlled trial. Journal of Medical Internet Research 2007; 9:e7.

32. Marcus BH, Forsyth LH. (2003) Motivating people to be physically active. Champaign: Human Kinetics.

33. Marcus BH, Lewis BA. Physical activity and the stage of motivational readiness for change model. Research Digest 2003; 4:1-8.

34. Maibach EW, Cotton D. (1995) Motivating people to behavior change: a stage social cognitive approach to message design. In Maibach E, Oarrott RL, eds. Designing health messages. Approaches from communication theory and public health practice. Sage, pp. 41.

35. Marcus BH, Owen N. Motivational readiness, self-efficacy and decision-making for exercise. Journal of Applied Social Psychology 1992; 22:3-16.

36. De Bourdeaudhuij I, Philippaerts R, Crombez G, et al. Stages of change for physical activity in a community sample of adolescents. Health Education Research 2005; 20: 357-366.

37. Blair S, Dunn AL, Marcus BH, et al. (2001) Active living every day. Human Kinetics.

38. Marcus BH, Taylor ER, Bock BC, et al. (1998) Jump start to health manual: the Center for behavorial and preventive medicine. The Miriam Hospital.

39. Canadian Society for Exercise Physiology. PAR-Q and You. www.csep.ca/cmfiles/publications/parq/par-q.pdf (accessed Feb. 20, 2012).

40. Linstone H, Turoff M. (1975) The Delphi method: techniques and applications. Reading, MA: Adison-Wesley.

41. Franklin VL, Waller A, Pagliari C, et al. A randomized controlled trial of sweet talk, a text-messaging system to support young people with diabetes. Diabetic Medicine 2006; 23:1332-1338.

42. Neville R, Greene A, MeLeod J, et al. Mobile phone text messaging can help young people manage asthma. British Medical Journal 2002; 325:600.

43. Obermayer JL, Riley WT, Asif O, et al. College smokingcessation using cell phone text messaging. Journal of American College Health 2004; 53:71.

44. Riley W, Obermayer J, Jean-Mary J. Internet and mobile phone text messaging intervention for college smokers. Journal of American College Health 2008; 57:245-248.

45. Crocker RE, Bailey D, Faulkner RA, et al. Measuring general levels of physical activity: preliminary evidence for the Physical Activity Questionnaire for older children. Medicine Science in Sport and Exercise 1997; 29:1344-1349. 
46. Kowalski KC, Crocker RE, Faulkner RA. Validation of the physical activity questionnaire for older children. Pediatrics Exercise Science 1997; 9:174-186.

47. Smeets T, Brug J, de Vries H. Effects of tailoring health messages on physical activity. Health Education Research 2008; 23:402-413.

48. Crutzen R, de Nooijer J, Candel MJJM, et al. Adolescents who intend to change multiple health behaviours choose greater exposure to an Internet-delivered intervention. Journal of Health Psychology 2008; 13:906-911.

49. Schwarzer R. Modeling health behavior change: how to predict and modify the adoption and maintenance of health behavior. Applied Psychology 2008; 57:1-29.

50. Sundar SS. (2007) Social psychology of interactivity in human-website interaction. In Joinson $\mathrm{AN}$, et al., eds. The Oxford handbook of Internet psychology. Oxford, New York: Oxford University Press.

51. Kiousis S. Interactivity: a concept explication. New Media and Society 2002; 4:355-383.

52. Waller A, Franklin V, Pagliari C, et al. Participatory design of a text message scheduling system to support young people with diabetes. Health Informatics Journal 2006; 12: 304-318.

53. Davis F, Bagozzi PR, Warshaw PR. User acceptance of computer technology: a comparison of two theoretical model. Management Science 1989; 35:982-1003.

54. Curry SJ. eHealth research and healthcare delivery: beyond intervention effectiveness. American Journal of Preventive Medicine 2007; 32:S127-S130.

55. Glasgow RE. eHealth evaluation and dissemination research. American Journal of Preventive Medicine 2007; 32: S119-S126.

Address correspondence to: Dr. Patrick W.C. Lau Department of Physical Education

Hong Kong Baptist University DLB 643, David C. Lam Bldg, HKBU Kowloon Tong, Hong Kong

E-mail:wclau@hkbu.edu.hk

\section{Appendices}

Appendix 1

Interface of Teenstep.com

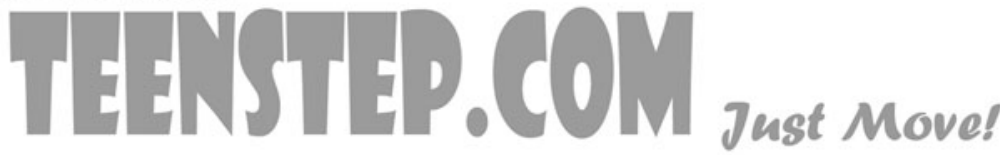

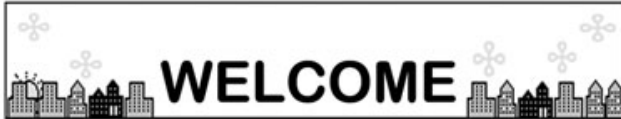

主頁

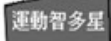

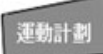
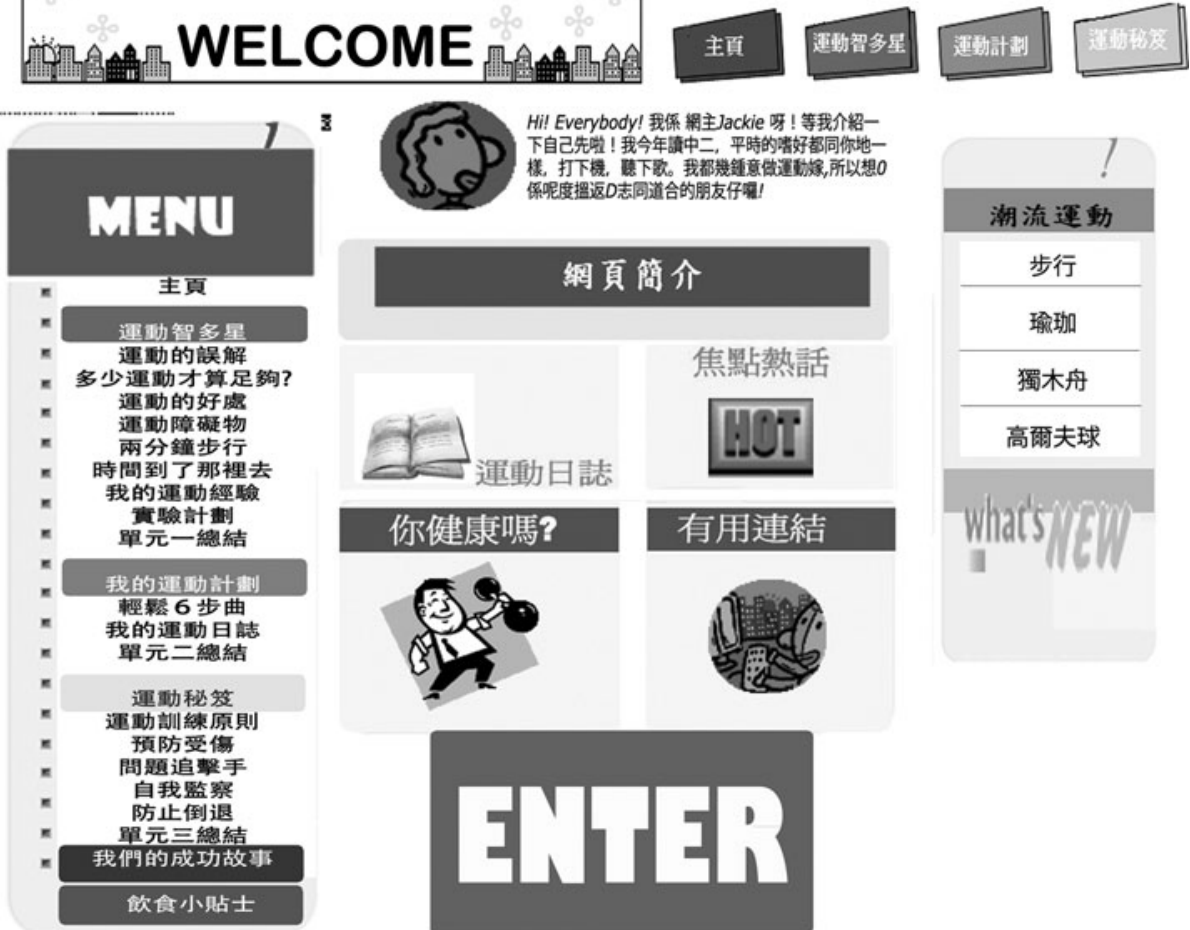

Hi! Everybody! 我係綡主Jackie 呀! 等我介紹一

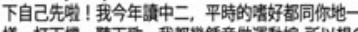

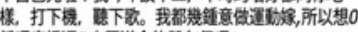

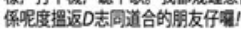
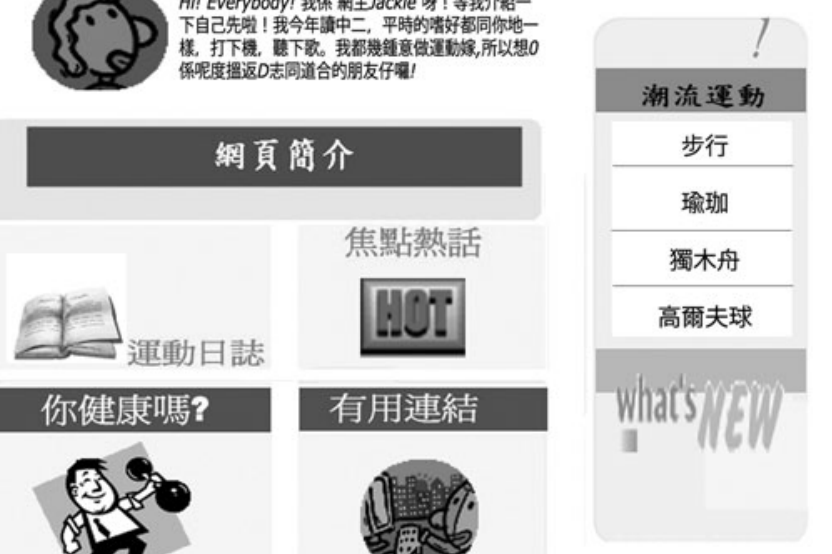

你健康嗎?

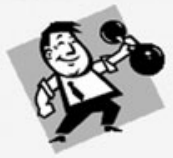

有用連結

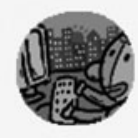


Appendix 2

Content of Teenstep.com

\begin{tabular}{ll}
\hline Content of Teenstep.com \\
\hline Module 1: Why Active?
\end{tabular}

\subsection{Misconception of PA}

The correct concept of "physical activity". It included the definition, types, and recommendations suggested by the International PA guidelines.

\subsection{Benefits and barrier of $P A$}

The pros and cons of engaging in PA. Common PA benefits and barriers and solutions for overcoming the PA barriers were provided.

\subsection{Take a small step}

Module 2: Becoming Active

Implementation of simple PA tasks to create successful experience that encourages PA behaviour change.

\subsection{Exploring PA opportunities}

Evaluation of time spent in sedentary behaviour and PA. It also provided suggestions for replacing sedentary activities with $\mathrm{PA}$.

\subsection{My PA experience}

Revision of previous successful and failure PA experiences. Participants were encouraged to review how these experiences affect their current PA. Suggestions for minimizing the negative impact of failure experiences were provided.

\subsection{Goal setting strategies}

Module 3: Set Goals!

A 6-step goal setting strategy was illustrated. It also included the following components: Needs assessment; SMART principles, sample activity programs (i.e. walking, weight loss, stretching and strength training) and progress evaluation.

\subsection{Physical activity planner}

APA scheduler was established for PA goal setting and daily PA self-monitoring.

\subsection{Relapse prevention}

$$
\text { Module 4: Keep Going! }
$$

Understanding high risk situations and incorporating the I-D-E-A coping strategies.

\subsection{Self-monitoring}

Self-monitoring skills (i.e. time and stress management) and tools (heart rate monitor and pedometer) were presented-- which assisted long-term PA behaviour change.

\subsection{Successful stories}

Others

An online chat room was developed for participants to seek advice from experts and share their successful PA experiences with other participants.

5.2 Trendy sports activities

Introduction to trendy sports for teens.

\subsection{Healthy eating}

Information about healthy diet and instructions for reading food label.

5.4 Useful link

Hyperlink to other credible websites such as World Health Organization (WHO), Centres for Disease Control, Health Department of Hong Kong Special Administration Region (HKSAR) and Leisure \& Cultural Service Department of HKSAR were provided.

\subsection{What's new}

Website update, announcement for monthly incentive winner. 
Appendix 3

Sample messages of the SMS motivators

Message type Examples

1. Motivational

"How was your day?" Jackie"

"Hey, you are great, how you maintain your exercise habit? Jackie"

2. Informational

"I updated the website, let's check it out! Jackie"

"Remember to set your weekly PA goal. Jackie"

3. Behavioral skills

"Don't use the remote control, move to change the TV channel. Jackie"

"Refill your water bottle at a farther drinking fountain! Jackie"

4. Reinforcement for

Benefit (outlook):

PA benefits

"Physical activity helps you maintain good posture, you look smart!

Jackie"

5. Solution for PA

Example barrier: I did not have time to exercise

barriers

Solution for PA barriers presented in stage-matched voice tones:

- pre-contemplation

"I am too busy to exercise too, so I make use of the staircase in the subway station. Well, less is better than zero! Hope it helps! Jackie"

- Contemplation

"Let's make use of the staircase in the subway station if you are too busy to exercise, what do you think about it? Jackie"

- Preparation

"Just take the staircase in the subway station if you are too busy to exercise. Jackie"

Note: The original SMS motivators were written in Cantonese. 